\title{
Images of the month: Intractable vomiting and neuromyelitis optica spectrum disorder
}

\author{
Authors: Seng Wee Cheo, ${ }^{\mathrm{A}}$ Qin Jian Low, ${ }^{\mathrm{B}}$ Yee Ann $\operatorname{Tan}^{\mathrm{C}}$ and Yuen Kang Chia
}

\begin{abstract}
Neuromyelitis optica spectrum disorder (NMOSD) is a rare inflammatory disorder of the nervous system which can be potentially debilitating. Its prevalence is estimated to be around $0.5-10$ per 100,000 population with predilection towards Asians and females. It can be diagnosed based on core clinical characteristics, serum aquaporin antibodies and neuroimaging features. It is important to pick up the diagnosis of NMOSD as the treatment is different from other demyelinating disease. Here, we illustrate a case of NMOSD presented with intractable vomiting.
\end{abstract}

KEYWORDS: Neuromyelitis optica spectrum disorder, NMOSD, vomiting, inflammatory disorder, neuroimaging

DOI: $10.7861 /$ clinmed.2020-0019

\section{Case presentation}

A 44-year-old woman with no past illness presented with a 2-week history of intractable vomiting, vertigo and diplopia. She then developed generalised body weakness and bulbar weakness. Examinations showed non-conforming multiple cranial neuropathies. Subsequently, she developed type 2 respiratory failure and required mechanical ventilation.

Her workup showed positive serum aquaporin-4 (AQP4) antibodies and positive anti-nuclear antibodies. Her magnetic resonance imaging (MRI) of the brain showed lesions of the periventricular region at the 4 th ventricle, extending to dorsal midbrain, pons and dorsal medulla (Fig 1). She was diagnosed to have neuromyelitis optica spectrum disorder (NMOSD) based on positive AQP4 and one core clinical characteristic - area postrema syndrome. She was then given pulses of methylprednisolone and therapeutic plasma exchange.

Her course of hospitalisation was complicated by aspiration pneumonia due to bulbar weakness. She was treated with antibiotics and responded positively. After five cycles of

Authors: A physician, Hospital Lahad Datu, Lahad Datu, Malaysia; B physician, Hospital Sultanah Nora Ismail, Batu Pahat, Malaysia; Cphysician, Hospital Queen Elizabeth, Kota Kinabalu, Malaysia; ${ }^{D}$ consultant neurologist, Hospital Queen Elizabeth, Kota Kinabalu, Malaysia therapeutic plasma exchange, she showed good clinical improvement. She was then started on maintenance azathioprine to prevent relapse and put on maintenance therapeutic plasma exchange. She recovered well and was discharged with Expanded Disability Status Scale of 2.0.

\section{Discussion}

NMOSD is an inflammatory central nervous system (CNS) disorder that is distinct from multiple sclerosis. It is an autoimmune demyelinating disorder for which the AQP4 water channels are the major target antigen. ${ }^{1}$ The understanding of NMOSD has largely evolved since the novel discovery of serum AQP 4 antibodies. Since the introduction of revised diagnostic criteria for NMOSD in 2015, NMOSD can be diagnosed based on serum AQP4-immunoglobulin $\mathrm{G}$, core clinical characteristics and neuroimaging features. ${ }^{2}$

The core clinical characteristics include optic neuritis, acute myelitis, area postrema syndrome, acute brainstem syndrome, symptomatic narcolepsy or diencephalic syndrome, and symptomatic cerebral syndrome. Clinically, the presentation of a patient with NMOSD depends on the area attacked by the antibodies. Patients with acute myelitis may present with paraplegia, tetraplegia with a sensory level. Area postrema syndrome results in intractable hiccoughs and vomiting. Acute brainstem syndrome can present with multiple cranial nerve palsies. $^{3}$

MRI of the brain remains the mainstay of neuroimaging for NMOSD. MRI abnormalities are often located at areas with high AQP4 expression. MRI brain findings can be classified into periependymal lesions surrounding the ventricular system, hemispheric white matter lesions, lesions involving corticospinal tracts, and non-specific and enhancing lesions. Periependymal lesions involve the third ventricle, fourth ventricle, lateral ventricles and cerebral aqueduct. One of the most specific MRI brain lesions in NMOSD is a lesion in the dorsal brainstem adjacent to the fourth ventricle. $^{4}$

The treatment of NMOSDs can be broadly divided into acute treatment and preventive therapy. The aim of acute treatment is to suppress the acute inflammatory attack, minimize CNS damage and improve long-term neurological outcome. Typically, highdose methylprednisolone is given as acute therapy for NMOSD flare followed by maintenance steroids. Plasma exchange can be considered in patients with inadequate response to steroids. ${ }^{5}$ In terms of preventive therapy, azathioprine, mycophenolate mofetil, rituximab and, recently, eculizumab can be offered to patients. 


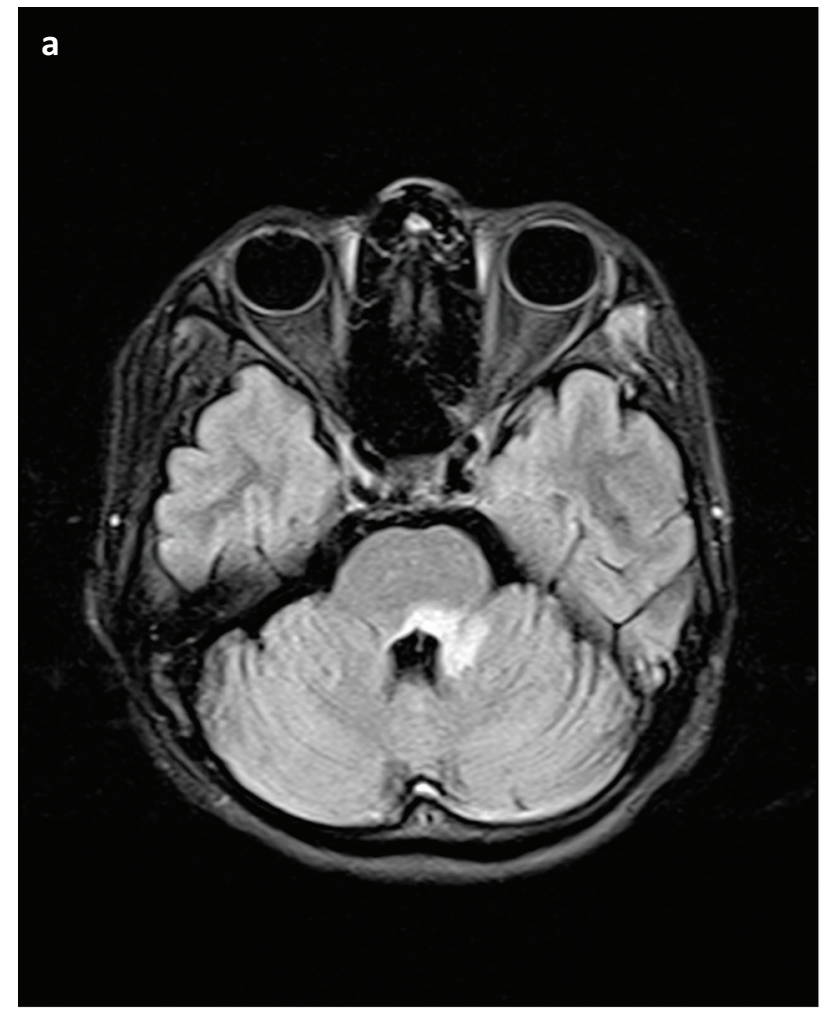

\section{References}

1 Wingerchuk DM, Banwell B, Bennett JL et al. International consensus diagnostic criteria for neuromyelitis optica spectrum disorders. Neurology 2015;85:177-89.

2 Dutra BG, da Rocha AJ, Nunes RH, Maia ACMJr. Neuromyelitis optica spectrum disorders: Spectrum of MR imaging findings and their differential diagnosis. Radiographics 2018;38:169-93.

3 Huda S, Whittam D, Bhojak M et al. Neuromyelitis optica spectrum disorders. Clin Med 2019:19:169-76.

$4 \mathrm{Kim} \mathrm{HJ}$, Paul F, Lana-Peixoto MA et al. MRI characteristics of neuromyelitis optica spectrum disorder: an international update. Neurology 2015;84:1165-73.

5 Kessler RA, Mealy MA, Levy M. Treatment of neuromyelitis optica spectrum disorder: Acute, preventive, and symptomatic. Curr Treat Options Neurol 2016;18:2.

Address for correspondence: Dr Seng Wee Cheo, Department of Internal Medicine, Hospital Lahad Datu, Peti Bersurat 60065, 91110, Lahad Datu, Sabah, Malaysia.

Email: cheosengwee@gmail.com

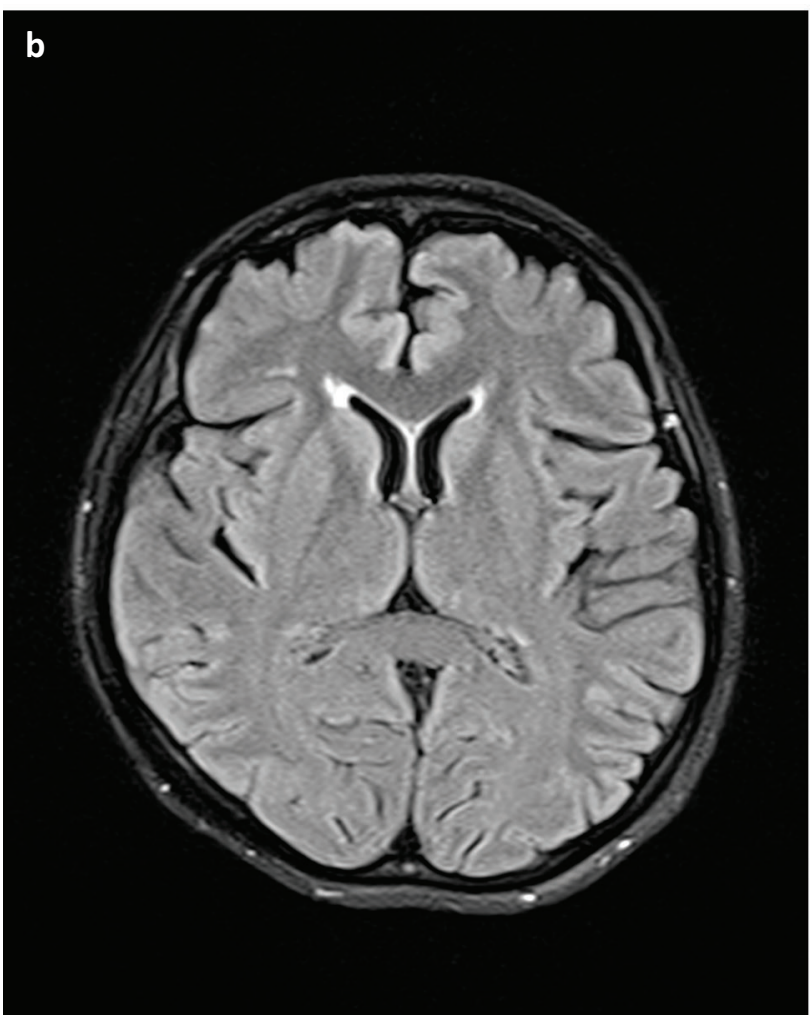

Fig 1. Magnetic resonance of the brain showing lesions (bright area) of the periventricular region at the 4 th ventricle, extending to dorsal midbrain, pons and dorsal medulla. a) Fourth ventricle. b) Lateral ventricles. 